\title{
Quantum Simulation of the Majorana Equation and Unphysical Operations
}

\author{
J. Casanova, ${ }^{1}$ C. Sabín, ${ }^{2}$ J. León, ${ }^{2}$ I. L. Egusquiza, ${ }^{3}$ R. Gerritsma, ${ }^{4,5}$ C. F. Roos, ${ }^{4,5}$ J. J. García-Ripoll, ${ }^{2}$ and E. Solano ${ }^{1,6}$ \\ ${ }^{1}$ Departamento de Química Física, Universidad del País Vasco - Euskal Herriko Unibertsitatea, Apdo. 644, 48080 Bilbao, Spain \\ ${ }^{2}$ Instituto de Física Fundamental, CSIC, Serrano 113-bis, 28006 Madrid, Spain \\ ${ }^{3}$ Departamento de Física Teórica, Universidad del País Vasco - Euskal Herriko Unibertsitatea, Apdo. 644, 48080 Bilbao, Spain \\ ${ }^{4}$ Institut für Quantenoptik und Quanteninformation, Österreichische Akademie der Wissenschaften, \\ Otto-Hittmair-Platz 1, A-6020 Innsbruck, Austria \\ ${ }^{5}$ Institut für Experimentalphysik, Universität Innsbruck, Technikerstrasse 25, A-6020 Innsbruck, Austria \\ ${ }^{6}$ IKERBASQUE, Basque Foundation for Science, Alameda Urquijo 36, 48011 Bilbao, Spain
}

(Received 22 April 2011; published 9 December 2011)

\begin{abstract}
We design a quantum simulator for the Majorana equation, a non-Hamiltonian relativistic wave equation that might describe neutrinos and other exotic particles beyond the standard model. Driven by the need of the simulation, we devise a general method for implementing a number of mathematical operations that are unphysical, including charge conjugation, complex conjugation, and time reversal. Furthermore, we describe how to realize the general method in a system of trapped ions. The work opens a new front in quantum simulations.
\end{abstract}

DOI: 10.1103/PhysRevX.1.021018

Subject Areas: Quantum Physics, Optics

A quantum simulator is a device engineered to reproduce the properties of an ideal quantum model. This stillemerging topical area has generated a remarkable exchange of scientific knowledge between apparently unconnected subfields of physics. In terms of applications, it allows for the study of quantum systems that cannot be efficiently simulated on classical computers [1]. While a quantum computer could in principle implement a universal quantum simulator [2], only particular systems have been simulated up to now using dedicated quantum simulators [3]. Still, there is a wealth of successful cases, such as spin models [4,5], quantum chemistry [6], and quantum phase transitions [7]. The quantum simulation of fermionic systems [8] and relativistic quantum physics have also attracted recent attention, reproducing dynamics and effects that are currently out of experimental reach. Examples include black holes in Bose-Einstein condensates [9], quantum field theories [10,11], and recent quantum simulations of relativistic quantum effects such as Zitterbewegung and the Klein paradox [12-16] in trapped ions.

In this paper, we show how the Majorana equation [17] can be simulated in an analog quantum simulator, having the implementation of complex conjugation of the wave function as a key requirement. In this manner, we are able to propose a general scheme for implementing this and other unphysical operations, such as charge conjugation and time reversal. The implementations constitute a novel toolbox of accessible quantum operations in the general frame of quantum simulations. While quantum simulators

Published by the American Physical Society under the terms of the Creative Commons Attribution 3.0 License. Further distribution of this work must maintain attribution to the author(s) and the published article's title, journal citation, and DOI. may soon realize calculations that are impossible for classical computers, we show here the possibility of implementing quantum dynamics that do not occur in the real space-time quantum world.

The Majorana equation is a relativistic wave equation for fermions where the mass term contains the charge conjugate of the spinor, $\psi_{c}$,

$$
i \hbar \not \psi=m c \psi_{c} .
$$

Here, $\not \supset=\gamma^{\mu} \partial_{\mu}$ and $\gamma_{\mu}$ are the Dirac matrices [18], while the non-Hamiltonian character stems from the simultaneous presence of $\psi$ and $\psi_{c}$. The significance of the Majorana equation lies in the fact that it can be derived from first principles in a similar fashion as the Dirac equation $[17,19]$. Both wave equations are Lorentz invariant but the former preserves helicity and does not admit stationary solutions. The Majorana equation is considered a possible model [20] for describing exotic particles in supersymmetric theories-photinos and gluinos-or in grand unified theories, as with the case of neutrinos. Indeed, the discussion of whether neutrinos are Dirac or Majorana particles still remains open [21]. Despite the similar naming, however, this work is neither related to the Majorana fermions (modes) in many-body systems $[22,23]$, nor to the Majorana fermions (spinors) in the Dirac equation [20,24].

In order to simulate the Majorana equation, we have to solve a fundamental problem: the physical implementation of antilinear and antiunitary operations in a quantum simulator. Here, we introduce a mapping [25] by which complex conjugation, an unphysical operation, becomes a unitary operation acting on an enlarged Hilbert space. The mapping works in arbitrary dimensions and can be immediately applied on different experimental setups. We show how to simulate the Majorana equation 
in $1+1$ dimensions and other unphysical operations using two trapped ions and their interactions with laser pulses. We also give a recipe for measuring observables and a road map toward more general scenarios. In this sense, this work provides a novel toolbox for quantum simulations.

There are three discrete symmetries [26] that are central to quantum mechanics and our understanding of particles, fields, and their interactions: parity $\mathcal{P}$, time reversal $\mathcal{T}$, and charge conjugation $\mathcal{C}$. None of these operations can be carried out in the real world: $\mathcal{P}$ involves a global change of physical space, while $\mathcal{C}$ and $\mathcal{T}$ are antiunitaries. However, there is no apparent fundamental restriction for implementing them in physical systems that simulate quantum mechanics. We will focus on the study of antiunitary operations, which can be decomposed into a product of a unitary, $\mathcal{U}_{\mathcal{C}}$ or $\mathcal{U}_{\mathcal{T}}$, and complex conjugation, $\mathcal{K} \psi=\psi^{*}$. We consider the mapping of the quantum states of an $n$-dimensional complex Hilbert space $\mathbb{C}_{n}$ onto a real Hilbert space [25] $\mathbb{R}_{2 n}$,

$$
\psi \in \mathbb{C}_{n} \rightarrow \Psi=\frac{1}{2}\left(\begin{array}{c}
\psi+\psi^{*} \\
i\left(\psi^{*}-\psi\right)
\end{array}\right) \in \mathbb{R}_{2 n} .
$$

This mapping can be implemented by means of an auxiliary two-level system, such that $\mathbb{R}_{2 n} \in \mathcal{H}_{2} \otimes \mathcal{H}_{n}$. In this manner, the complex conjugation of the simulated state becomes a local unitary $V_{\mathcal{K}}$ acting solely on the ancillary space, $\mathcal{K} \psi=\psi^{*} \rightarrow V_{\mathcal{K}} \Psi=\left(\sigma_{z} \otimes \mathbb{1}\right) \Psi$, and thus is physically implementable for a wave function of arbitrary dimensions. Furthermore, unitaries and observables can also be mapped onto the real space, $O \rightarrow \Theta=$ $\mathbb{1} \otimes O_{r}-i \sigma^{y} \otimes O_{i}$, where $O_{r}=\frac{1}{2}(O+\mathcal{K} O \mathcal{K})$ and $O_{i}=$ $-\frac{i}{2}(O-\mathcal{K} O \mathcal{K})$, preserving unitarity and Hermiticity. The proposed simulator also accommodates the antiunitary operations $\mathcal{C}=\mathcal{U}_{\mathcal{C}} \mathcal{K}$ and $\mathcal{T}=\mathcal{U}_{\mathcal{T}} \mathcal{K}$. To this end, we have to choose a particular representation that fixes the unitaries $\mathcal{U}_{\mathcal{C}}$ and $\mathcal{U}_{\mathcal{T}}$, as will be shown below.

We possess now the basic tools to simulate the Majorana equation (1). The expression for the charge-conjugate spinor is given by $\psi_{c}=\mathcal{W} \gamma^{0} \mathcal{K} \psi$, with $\mathcal{W}$ a unitary matrix satisfying $\mathcal{W}^{-1} \gamma^{\mu} \mathcal{W}=-\left(\gamma^{\mu}\right)^{T}$. We illustrate now the proposed quantum simulation with the case of $1+1$ dimensions. Here, a suitable representation of charge conjugation is $\psi_{c}=i \sigma_{y} \sigma_{z} \psi^{*}$, for which $\mathcal{W}=i \sigma_{y}$, and the Majorana equation reads

$$
i \hbar \partial_{t} \psi=c \sigma_{x} p_{x} \psi-i m c^{2} \sigma_{y} \psi^{*},
$$

where $p_{x}=-i \hbar \partial_{x}$ is the momentum operator. In particular, our mapping of Eq. (2) for $n=2$ reads

$$
\left(\begin{array}{l}
\psi_{1} \\
\psi_{2}
\end{array}\right) \in \mathbb{C}_{2} \rightarrow \Psi=\left(\begin{array}{c}
\psi_{1}^{r} \\
\psi_{2}^{r} \\
\psi_{1}^{i} \\
\psi_{2}^{i}
\end{array}\right) \in \mathbb{R}_{4},
$$

where $\psi_{1,2}^{r, i}$ are the real and imaginary parts of $\psi_{1}$ and $\psi_{2}$, respectively. Surprisingly, the non-Hermitian Majorana equation for a complex spinor becomes a $3+$ 1-dimensional Dirac equation for a four-component real bispinor,

$$
i \hbar \partial_{t} \Psi=\left[c\left(\mathbb{1} \otimes \sigma_{x}\right) p_{x}-m c^{2} \sigma_{x} \otimes \sigma_{y}\right] \Psi,
$$

with dimensional reduction, $p_{y}, p_{z}=0$. Here, the dynamics preserves the reality of the bispinor $\Psi$ and cannot be reduced to a single $1+1$ Dirac particle. In general, the complex-to-real map in arbitrary space-time dimensions transforms a Majorana equation into a higher dimensional Dirac equation [27]. Since Eq. (5) is a Hamiltonian equation, it can be simulated in a quantum system.

The mapping of wave functions into higher-dimensional spinors also allows us to explore exotic symmetries and unphysical operations, which are otherwise impossible in nature. From Eqs. (3)-(5), for the 1 + 1-dimensional case, we can deduce that charge conjugation is implemented in the enlarged space via the unitary operation $V_{\mathcal{C}}$,

$$
\psi_{c}=\mathcal{C} \psi=\mathcal{U}_{C} K \psi \rightarrow V_{\mathcal{C}} \Psi=-\left(\sigma_{z} \otimes \sigma_{x}\right) \Psi .
$$

We can perform something similar with time reversal, defined as the change $t \rightarrow(-t)$. In this case, we expect [19] $i \hbar \partial_{\tau} \psi^{\prime}(\tau)=H \psi^{\prime}(\tau)$, where the time variable $\tau=-t$ and the modified spinor $\psi^{\prime}(\tau)=\mathcal{T} \psi(t)$. In order to preserve scalar products and distances, the time-reversal operator must be an antiunitary operator and thus decomposable as the product $\mathcal{T}=\mathcal{U}_{\mathcal{T}} \mathcal{K}$. In $1+1$ dimensions, imposing that the Hamiltonian be invariant under time reversal $\mathcal{T}^{-1} H \mathcal{T}$ implies that the unitary operator satisfies $\mathcal{U}_{\mathcal{T}}^{-1}\left(i \sigma_{x} \partial_{x}\right) \mathcal{U}_{\mathcal{T}}=-i \sigma_{x} \partial_{x}$, with a possible choice being $U_{\mathcal{T}}=\sigma_{z}$. In other words, in the enlarged simulation space

$$
\mathcal{T} \psi=\mathcal{U}_{\mathcal{T}} \mathcal{K} \psi \rightarrow V_{\mathcal{T}} \Psi=\left(\sigma_{z} \otimes \sigma_{z}\right) \Psi .
$$

Figure 1 illustrates a scheme of the simulated symmetries. As mentioned before, quantum simulations of unphysical operations can be straightforwardly extended to higher dimensions. In this sense, Eqs. (6) and (7) will be valid for wave functions $\psi$ in a $d$-dimensional space as long as we consider the complex conjugation of an arbitrary wave function as $V_{\mathcal{K}} \Psi=\left(\sigma_{z} \otimes \mathbb{1}_{d}\right) \Psi$.

The proposed protocol for implementing unphysical operations within a physical setup allows us to deal with situations that are, otherwise, intractable with conventional quantum simulations. To exemplify the value of this novel building block in the quantum-simulation toolbox, we consider a case of an advanced quantum simulation, where after a certain time of unitary (physical) evolution of the system, an unphysical operation, such as the charge conjugate or time reversal, needs to be performed, before the unitary evolution is continued. Such a situation is impossible to reproduce seamlessly with classical computers. 


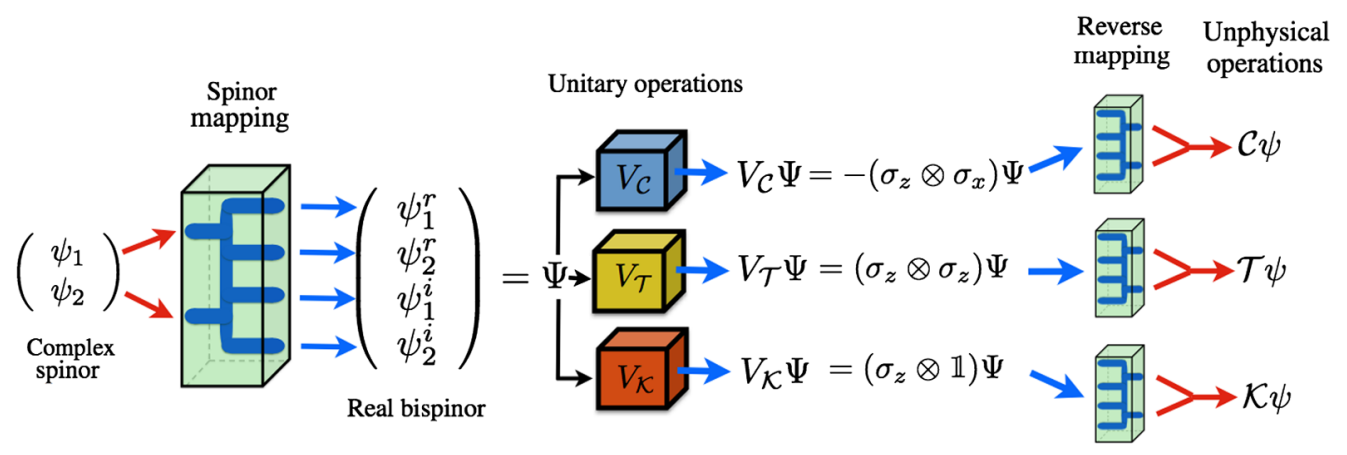

FIG. 1. Diagram showing the different steps involved in the proposed quantum simulation of unphysical operations in $1+1$ dimensions.

With tools that currently exist in quantum simulations, we would need to stop the dynamics, implement a full quantum tomography of the current quantum state associated to a huge Hilbert space, apply the unphysical operation in a classical computer, encode back the modified quantum state into the experimental setup, and then to proceed with the quantum simulation. Clearly, this task would be impossible with classical resources but could be accomplished with a suitable implementation of our proposed ideas.

In a recent experiment, the dynamics of a free Dirac particle was simulated with a single trapped ion [13]. Here, Eq. (5) has a more complex structure requiring a different setup. Moreover, the encoded Majorana dynamics requires a systematic decoding via a suitable reverse mapping of observables. We can simulate Eq. (5) with two trapped ions, with lasers coupling their internal states and motional degrees of freedom. The kinetic part, $c p_{x}\left(\mathbb{1} \otimes \sigma_{x}\right)$, is created with a laser tuned to the blue and red motional sidebands of an electronic transition [12,14], which is focused on ion 2. The second term, $\sigma_{x} \otimes \sigma_{y}$, is derived from detuned red and blue sideband excitations stimulated in each ion. The Hamiltonian describing this situation reads as

$$
\begin{aligned}
H= & \hbar \frac{\omega_{0}}{2} \sigma_{1}^{z}+\hbar \frac{\omega_{0}}{2} \sigma_{2}^{z}+\hbar \nu a^{\dagger} a+\hbar \nu_{r} b^{\dagger} b \\
& +\hbar \Omega\left\{\left[e^{i\left(q z_{1}-\omega_{1} t+\phi_{1}\right)}+e^{i\left(q z_{1}-\omega_{1}^{\prime} t+\phi_{1}^{\prime}\right)}\right] \sigma_{1}^{+}+\text {H.c. }\right\} \\
& +\hbar \Omega\left\{\left[e^{i\left(q z_{2}-\omega_{2} t+\phi_{2}\right)}+e^{i\left(q z_{2}-\omega_{2}^{\prime} t+\phi_{2}^{\prime}\right)}\right] \sigma_{2}^{+}+\text {H.c. }\right\} \\
& +\hbar \tilde{\Omega}\left\{\left[e^{i\left(q z_{2}-\omega t+\phi\right)}+e^{i\left(q z_{2}-\omega^{\prime} t+\phi^{\prime}\right)}\right] \sigma_{2}^{+}+\text {H.c. }\right\} .
\end{aligned}
$$

Here $z_{1,2}=Z \pm z / 2$ are the positions, of the two ions, respectively, measured from their center of mass $Z$ and relative coordinate $z$. The phases of the lasers $\phi_{i}$, for $i=1,2\left(\phi, \phi^{\prime}\right)$, are controlled to simulate the interaction term (kinetic term). The frequencies of the center-of-mass vibration and the stretch mode are given by $\nu$ and $\nu_{r}=\sqrt{3} \nu$, respectively, and whereas $a^{\dagger}, a, b^{\dagger}$, and $b$, are the corresponding creation and annihilation operators. Finally, $\Omega$ and $\tilde{\Omega}$ are the laser Rabi frequencies in the rotating-wave approximation. With the adequate choice of parameters, $\omega_{1}=\omega_{0}+\nu_{r}-\delta, \quad \omega_{1}^{\prime}=\omega_{0}-\nu_{r}+\delta, \quad \omega_{2}=\omega_{0}-\nu_{r}+\delta$,

$\omega_{2}^{\prime}=\omega_{0}+\nu_{r}-\delta, \quad \omega=\omega_{0}-\nu, \quad \omega^{\prime}=\omega_{0}+\nu, \quad \phi=\pi$,

$\phi^{\prime}=0, \quad \phi_{1}=\pi / 2, \quad \phi_{1}^{\prime}=\pi / 2, \quad \phi_{2}=0, \quad \phi_{2}^{\prime}=0$,

the Hamitonian in the interaction picture reads as

$$
\begin{aligned}
H= & \hbar \eta_{r} \Omega\left(\sigma_{x} \otimes \mathbb{1}-\mathbb{1} \otimes \sigma_{y}\right)\left(b^{\dagger} e^{i \delta t}+b e^{-i \delta t}\right) \\
& +\hbar \eta \tilde{\Omega}\left(\mathbb{1} \otimes \sigma_{x}\right) i\left(a^{\dagger}-a\right),
\end{aligned}
$$

where $\eta \equiv \eta_{r} 3^{1 / 4} \equiv \sqrt{\hbar / 4 m^{\prime} \nu} \ll 1$ is the Lamb-Dicke parameter and $m^{\prime}$ is the ion mass. In the limit of large detuning, we have $\delta \gg \eta_{r} \Omega \sqrt{\left\langle b^{\dagger} b\right\rangle}, \eta \tilde{\Omega}\left|\left\langle a^{\dagger}-a\right\rangle\right|$, and we recover Eq. (5) with the momentum operator $p_{x}=$ $i \hbar\left(a^{\dagger}-a\right) / 2 \Delta$ and the equivalences $c=2 \eta \Delta \tilde{\Omega}$ and $m c^{2}=2 \hbar \eta_{r}^{2} \Omega^{2} / \delta$ with $\Delta=\sqrt{\hbar / 4 m^{\prime} \nu}$. Introducing the ratio $\gamma=\left|m c^{2} /\left\langle c p_{x}\right\rangle\right|$, with $\left.\gamma=\frac{2\left(\eta_{r} \Omega / \delta\right)^{2}}{\left|\left\langle i\left(a^{\dagger}-a\right)\right\rangle\right|(\eta \Omega / \Omega} / \delta\right)$, we see that it is possible to tune the numerator and denominator independently to preserve the dispersive regime, while exploring simultaneously the range from $\gamma \simeq 0$ (ultrarelativistic limit) to $\gamma \rightarrow \infty$ (nonrelativistic limit).

An interesting feature of the Majorana equation in $3+1$ dimensions is the conservation of helicity. Its reminiscent in $1+1$ dimensions is an observable called, hereafter, pseudohelicity $\Sigma=\sigma_{x} p_{x}$. This quantity is conserved in the 1+1-dimensional Majorana dynamics of Eq. (3) but not in the $1+1$-dimensional Dirac equation. We will use this observable to illustrate measurements on the Majorana wave function. The mapping for operators can be simplified if we are only interested in their expectation values. Reconstructing the complex spinor with the nonsquare matrix $\psi=M \Psi$ and $M=(\mathbb{1} i \mathbb{1})$, associated with Eqs. (4) and (5), we have $\langle O\rangle_{\psi}=\langle\psi|O| \psi\rangle=$ $\left\langle\Psi\left|M^{\dagger} O M\right| \Psi\right\rangle=:\langle\tilde{O}\rangle_{\Psi}$. Therefore, to obtain the pseudohelicity $\Sigma$, we have to measure

$$
\tilde{\Sigma}=M^{\dagger} \sigma_{x} p_{x} M=\left(\mathbb{1} \otimes \sigma_{x}-\sigma_{y} \otimes \sigma_{x}\right) \otimes p_{x}
$$



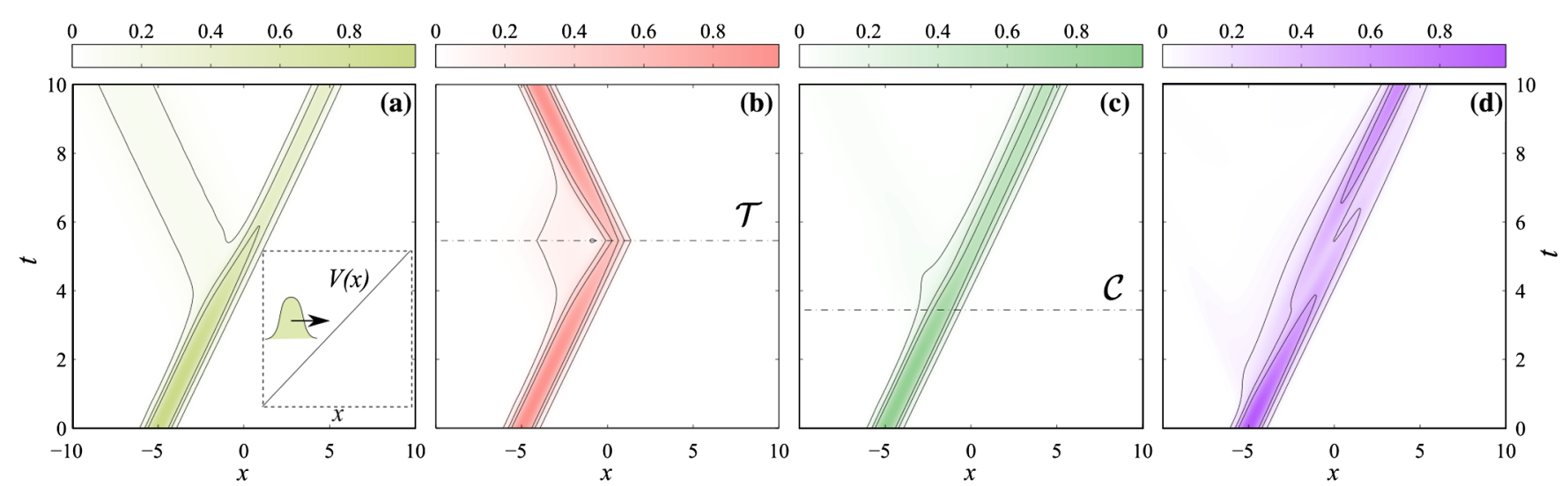

FIG. 2. Scattering of a fermion against a linearly growing potential (inset). (a) Ordinary Klein process. (b) At an instant of time indicated by the dashed line we apply the time-reversal operator $\mathcal{T}$ causing the particle to retrace its own trajectory. (c) Similar to (b) but now we apply charge conjugation, converting the particle into its antiparticle. (d) Scattering of a Majorana particle, which propagates through the potential. Parameters are $m=0.5, c=1$, and $V(x)=x$, in dimensionless units.

in the enlarged simulation space. In the type of trapped-ion experiments discussed above, we can use laser pulses to map information about the pseudohelicity onto the internal states. The application of a state-dependent displacement operation on ion $2, U_{2}=\exp \left[-i k\left(\mathbb{1} \otimes \sigma_{y}\right) \otimes p_{x} / 2\right]$, generated by resonant blue and red sidebands, followed by a measurement of $\mathbb{1} \otimes \sigma_{z}$, is equivalent to measuring the observable [13] $A(k)=\cos \left(k p_{x}\right)\left(\mathbb{1} \otimes \sigma_{z}\right)+\sin \left(k p_{x}\right)$ $\left(\mathbb{1} \otimes \sigma_{x}\right)$. Here, $k$ is proportional to the probe time $t_{\text {probe }}$. Note that $\left.\frac{d}{d k}\langle A(k)\rangle\right|_{k=0} \propto\left\langle\left(\mathbb{1} \otimes \sigma_{x}\right) \otimes p_{x}\right\rangle$. Therefore, the first term in (10) can be measured by applying a short probe pulse to the ions and measuring the initial slope of the observable $A(k)$. To measure the second term in Eq. (10), we apply the operation $U_{1}=\exp \left[-i k\left(\sigma_{x} \otimes \mathbb{1}\right) \otimes p_{x} / 2\right]$, and measure the spin correlation $\sigma_{z} \otimes \sigma_{x}$. We have, then, $\left.\frac{d}{d k}\left\langle\sigma_{z} \otimes \sigma_{x}\right\rangle\right|_{k=0}=2\left\langle\left(\sigma_{y} \otimes \sigma_{x}\right) \otimes p_{x}\right\rangle$.

So far, we have presented a complete toolbox for implementing unphysical operations, $\mathcal{C}, \mathcal{T}$, and $\mathcal{K}$. We can combine all these tools to study the dynamical properties of the transformed wave functions. To exemplify the kind of experiments that become available, we have studied the scattering of wave packets against a linearly growing potential, $V(x)=\alpha x$, with conventional numerical tools. It is known that repulsive potentials can be penetrated by Dirac particles [18] due to Klein tunneling [14,15]. This is shown in Fig. 2(a), where a Dirac particle splits into a fraction of a particle that bounces back, and a large antiparticle component that penetrates the barrier. This numerical experiment has been combined with the discrete symmetries and the Majorana equation. In Fig. 2(b) we apply the time-reversal operation after the particle has entered the barrier: All momenta are reversed and the wave packet is refocused, tracing back exactly its trajectory. In Fig. 2(c) we apply charge conjugation, changing the sign of the charge and turning a repulsive electric potential into an attractive one, which can be easily penetrated by the antiparticle. In Fig. 2(d), we show the scattering of a Majorana particle.
While there are no plane wave solutions in the Majorana equation, we can still see a wave packet penetrating the barrier, showing a counterintuitive insensitivity to the presence of it.

The previous implementation of discrete symmetries is valid both for Majorana and Dirac equations. Equally interesting is the possibility of combining both Dirac and

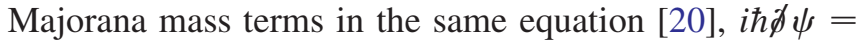
$m_{M} c \psi_{c}+m_{D} c \psi$, which still requires only two ions to implement experimentally. It also becomes feasible to have $C P$-violating phases in the Dirac mass term $m_{D} \exp \left(i \theta \gamma^{5}\right)$. Furthermore, we could study the dynamics of coupled Majorana neutrinos with a term $\bar{M} \psi_{c}$, where $\bar{M}$ is now a matrix and $\psi=\psi\left(x_{1}, x_{2}\right)$ is the combination of two such particles, simulated with three ions and two vibrational modes.

In summary, we have introduced a general method to implement quantum simulations of unphysical operations and non-Hamiltonian dynamics, such as the Majorana equation, in a Hamiltonian system.

The authors acknowledge funding from Basque Government Grants No. BFI08.211, IT559-10, and No. IT472-10; Spanish MICINN FIS2008-05705, FIS200910061, and FIS2009-12773-C02-01; QUITEMAD; the EC Marie-Curie program; and the CCQED and SOLID European projects.

[1] R. Feynman, Simulating Physics with Computers, Int. J. Theor. Phys. 21, 467 (1982).

[2] S. Lloyd, Universal Quantum Simulators, Science 273, 1073 (1996).

[3] I. Buluta and F. Nori, Quantum Simulators, Science 326, 108 (2009).

[4] H. Friedenauer, H. Schmitz, J. Glueckert, D. Porras, and T. Schätz, Simulating a Quantum Magnet with Trapped Ions, Nature Phys. 4, 757 (2008). 
[5] K. Kim, M.-S. Chang, S. Korenblit, R. Islam, E. E. Edwards, J. K. Freericks, G.-D. Lin, L.-M. Duan, and C. Monroe, Quantum Simulation of Frustrated Ising Spins with Trapped Ions, Nature (London) 465, 590 (2010).

[6] B. P. Lanyon, J. D. Whitfield, G. G. Gillet, M. E. Goggin, M. P. Almeida, I. Kassal, J. D. Biamonte, M. Mohseni, B. J. Powell, M. Barbieri, A. Aspuru-Guzik, and A. G. White, Towards Quantum Chemistry on a Quantum Computer, Nature Chem. 2, 106 (2010).

[7] M. Greiner, O. Mandel, T. Esslinger, T. W. Hänsch, and I. Bloch, Quantum Phase Transition from a Superfluid to a Mott Insulator in a Gas of Ultracold Atoms, Nature (London) 415, 39 (2002).

[8] J. Casanova, A. Mezzacapo, L. Lamata, and E. Solano, Quantum Simulation of Interacting Fermion Lattice Models in Trapped Ions, arXiv:1110.3730.

[9] O. Lahav, A. Itah, A. Blumkin, C. Gordon, S. Rinott, A. Zayats, and J. Steinhauer, Realization of a Sonic Black Hole Analog in a Bose-Einstein Condensate, Phys. Rev. Lett. 105, 240401 (2010).

[10] J. Casanova, L. Lamata, I. L. Egusquiza, R. Gerritsma, C. F. Roos, J. J. Garcia-Ripoll, and E. Solano, Quantum Simulation of Quantum Field Theories in Trapped Ions, arXiv:1107.5233 [Phys. Rev. Lett. (to be published)].

[11] S.P. Jordan, K. S. M. Lee, and J. Preskill, Quantum Algorithms for Quantum Field Theories, arXiv:1111.3633.

[12] L. Lamata, J. León, T. Schätz, and E. Solano, Dirac Equation and Quantum Relativistic Effects in a Single Trapped Ion, Phys. Rev. Lett. 98, 253005 (2007).

[13] R. Gerritsma, G. Kirchmair, F. Zähringer, E. Solano, R. Blatt, and C.F. Roos, Quantum Simulation of the Dirac Equation, Nature (London) 463, 68 (2010).

[14] J. Casanova, J. J. García-Ripoll, R. Gerritsma, C. F. Roos, and E. Solano, Klein Tunneling and Dirac Potentials in Trapped Ions, Phys. Rev. A 82, 020101(R) (2010).

[15] R. Gerritsma, B. P. Lanyon, G. Kirchmair, F. Zähringer, C. Hempel, J. Casanova, J. J. García-Ripoll, E. Solano,
R. Blatt, and C.F. Roos, Quantum Simulation of the Klein Paradox with Trapped Ions, Phys. Rev. Lett. 106, 060503 (2011).

[16] L. Lamata, J. Casanova, R. Gerritsma, C. F. Roos, J. J. García-Ripoll, and E. Solano, Relativistic Quantum Mechanics with Trapped Ions, New J. Phys. 13, 095003 (2011).

[17] E. Majorana, Teoria Simmetrica dell'Elettrone e del Positrone, Nuovo Cimento 14, 171 (1937).

[18] B. Thaller, The Dirac Equation (Springer-Verlag, Berlin, 1992).

[19] A. Zee, Quantum Field Theory in a Nutshell (Princeton University Press, Princeton, 2003).

[20] A. Aste, A Direct Road to Majorana Fields, Symmetry 2, 1776 (2010).

[21] C. Giunti and C. W. Kim, Fundamentals of Neutrino Physics and Astrophysics (Oxford University Press, Oxford, 2007).

[22] A. Y. Kitaev, Unpaired Majorana Fermions in Quantum Wires, Phys. Usp. 44, 131 (2001).

[23] A. Mezzacapo, J. Casanova, L. Lamata, and E. Solano, Topological Qubits with Majorana Fermions in Trapped Ions, arXiv:1111.5603.

[24] F. Wilczek, Majorana Returns, Nature Phys. 5, 614 (2009).

[25] Since the early times of quantum mechanics, several mappings that turn complex wave functions onto real ones have been proposed, generating different kinds of wave equations. In this sense, it is possible to turn the Majorana equation into a Klein-Gordon-type (see [20]), or into a Dirac equation; see J. Serpe, Two-Component Wave Equations, Phys. Rev. 76, 1538 (1949).

[26] R.F. Streater and A.S. Wightman, PCT, Spin and Statistics and All That (Princeton University Press, Princeton, 2000).

[27] Majorana equations in $2+1$ and $3+1$ dimensions are transformed into $3+1$ and $7+1$ Dirac equations. 